\title{
Efecto del manejo sobre la diversidad de árboles en vegetación secundaria en la Reserva de la Biosfera de Calakmul, Campeche, México
}

\author{
Ana María Báez-Vargas ${ }^{1 *}$, Ligia Esparza-Olguín¹, Eduardo Martínez-Romero², Susana Ochoa- \\ Gaona $^{3}$, Neptalí Ramírez-Marcial ${ }^{1}$ \& Noel Antonio González-Valdivia ${ }^{4}$ \\ 1. Departamento de Conservación de la Biodiversidad, El Colegio de la Frontera Sur, México; abaez@ecosur.edu.mx, \\ lesparza@ecosur.mx,nramirezm@ecosur.mx \\ 2. Investigación y Soluciones Socioambientales AC, México; iss.presidencia@gmail.com \\ 3. Departamento de Ciencias de la Sustentabilidad, El Colegio de la Frontera Sur, México; sochoa@ecosur.mx \\ 4. Departamento de Ingenierías, Instituto Tecnológico de Chiná, México; siankaan2003@gmail.com \\ * Correspondencia
}

$$
\text { Recibido 11-VIII-2015. C Corregido 10-VIII-2016. Aceptado 12-IX-2016. }
$$

\begin{abstract}
Effect of management on tree diversity in secondary vegetation in the Biosphere Reserve of Calakmul, Campeche, Mexico. The tropical dry forest (BTS) of Yucatan Peninsula has been managed for centuries, but the relationship between these management efforts and their effects on trees diversity has not been fully understood. The aim of this study was to evaluate the effect of forest management (thinning, harvesting and enrichment of species), in the structure of secondary vegetation derived from dry tropical forests, in two communities in Calakmul, Campeche, Southeast Mexico. We analyzed changes in the composition, species richness, species diversity, and structure in secondary vegetation subject to following types of management: (1) secondary vegetation with beekeeping management (MA), secondary vegetation with forest management (MF), natural secondary vegetation (SM) and tropical dry forest (BTS). The species composition was similar between secondary vegetation managed and unmanaged. On the other hand, between managed secondary vegetation and BTS there were differences in species composition. Species richness was not different between all conditions. MA showed the lowest species diversity and presented higher average density (5 $413 \pm 770.26$ ind.ha ${ }^{-1}$ ).MF had lowest average density (3 $289 \pm 1183.60$ ind.ha $^{-1}$ ). BTS showed the highest average basal area $(24.89 \pm 1.56$ $\mathrm{m}^{2}$.ha-1) regarding the other conditions. We concluded that is necessary to keep monitoring the managed areas to detect effects of management that may be adverse or favorable to conservation of floristic diversity of BTS. Rev. Biol. Trop. 65 (1): 41-53. Epub 2017 March 01.
\end{abstract}

Key words: beekeeping management, forest management, dry forest, structure, floristic composition.

Los bosques tropicales secos (BTS) (Miranda \& Hernández-X, 1963; Murphy \& Lugo, 1986), han sido transformados por actividades humanas, principalmente la agricultura y ganadería (Lawrence et al., 2007). En la Región de Calakmul, ubicada en el Sureste del estado de Campeche, México (Galindo-Leal, 1999), después de la agricultura de roza-tumba y quema (agricultura tradicional), se permite el establecimiento de vegetación secundaria durante la fase de descanso (Vester et al., 2007). Bajo este sistema, las tierras se cultivan por periodos de varios años (dos a cinco años), y con el fin de recuperar la fertilidad del suelo, se dejan sin cultivar permitiendo el desarrollo de vegetación secundaria para repetir un nuevo ciclo de aprovechamiento (periodos de 15 años o más; Beckerman, 1987). De la vegetación secundaria se obtiene leña, carbón, materiales para construcción, miel, curtientes, medicina, entre otros usos (Navarro-Martínez, Schmook, \& Martínez-Castillo, 2000; Jiménez-Osornio, Duran-García, Dupuy-Rada, \& González-Iturbe, 2010). 
En la región de Calakmul más del $45 \%$ de la población tiene niveles de pobreza extrema (CONEVAL, 2012) y el nivel de marginación de las localidades es alto (CONAPO, 2010). Como una alternativa productiva, los habitantes de las comunidades Puebla de Morelia y Kilómetro Ciento Veinte, pertenecientes a la Asociación Regional de Silvicultores (ARS) de Calakmul, A.C., comenzaron a realizar manejo forestal desde el 2011. Este manejo forestal se realiza en la vegetación secundaria derivada de bosque tropical seco, con el fin de obtener recursos que les puedan generar ingresos económicos.

Diversos estudios reportan que las áreas bajo manejo forestal presentan diferencias en la composición florística, de estructura y diversidad de especies arbóreas, respecto a sitios que no son manejados (Kumar \& Shahabuddin, 2005; Fujii, Kubota, \& Enoki, 2009).

Lo mismo sucede en las áreas donde se ha practicado la agricultura tradicional, en las que la composición florística, diversidad de especies y estructura de la vegetación disminuyen, debido a la historia de uso de los sitios, en las que se consideran la intensidad (duración de los ciclos de cultivo y períodos de descanso), la frecuencia de uso (número de ciclos de cultivo) y el uso de agroquímicos (Lawrence, 2004; Ochoa-Gaona, Hernández-Vázquez, de Jong, \& Gurri-García, 2007).

Se han realizado estudios en los BTS, enfocados a evaluar las características estructurales de la vegetación en áreas bajo extracción forestal en diferentes periodos (Oliveira-Filho, Carvalho, Vilela, Curi, \& Fontes, 2004); con diferentes gradientes de disturbios y variables ambientales (Gillespie, Grijalva, \& Farris, 2000; Kennard, Gould, Putz, Fredericksen, \& Morales, 2002). También se han analizado los BTS en diferentes estados sucesionales, con distintas variables edáficas y con historias de usos particulares (Kalacska et al., 2004; Leiva, Mata, Rocha, \& Gutiérrez-Soto, 2009).

El trabajo analiza si el manejo realizado en Calakmul, puede ser una alternativa viable actualmente y a futuro, que combine la actividad productiva con la conservación de los recursos forestales. Considerando que en México la extracción y comercialización de productos forestales maderables requiere de permisos oficiales, que significan un alto costo para las comunidades. El trabajo tiene dos objetivos: (1) caracterizar el manejo de vegetación secundaria en Calakmul y (2) comparar la composición florística, diversidad de especies y la estructura de las especies arbóreas, en las parcelas con manejo, sin manejo y en bosques tropicales secos.

La hipótesis planteada es que la composición de especies y la estructura, serán distintas en la vegetación secundaria con manejo apícola o forestal respecto a la vegetación secundaria sin manejo, con menor densidad, riqueza y diversidad de especies en áreas manejadas que en las no manejadas. Finalmente, el área basal de especies arbóreas será mayor en áreas manejadas que en áreas sin manejo, debido a la eliminación de árboles suprimidos que compiten por los nutrientes y luz, incrementando el crecimiento de los individuos remanentes.

\section{MATERIALES Y MÉTODOS}

Área de estudio: Este estudio se realizó en el período entre abril y agosto 2013, en las comunidades Puebla de Morelia (PM, 18³1'22" N - 8940’42” W) y Kilómetro Ciento Veinte (Km, 120-18 $31^{\prime} 13^{\prime \prime} \mathrm{N}$ $\left.89^{\circ} 41^{\prime} 51^{\prime \prime} \mathrm{W}\right)$ pertenecientes al municipio de Calakmul, Campeche, México (INEGI, 2010). El clima en la zona es cálido subhúmedo con lluvias en verano, con temperatura media anual de $24.4{ }^{\circ} \mathrm{C}$ y una precipitación media anual de $1012 \mathrm{~mm}$ (INEGI, 2012). Los suelos predominantes en esta área corresponden a: leptosoles $(34.93 \%)$, vertisoles $(33.75 \%)$, feozems $(24.93 \%)$, gleysoles $(4.44 \%)$ y fluvisoles (1.86\%; INEGI, 2009).

La vegetación original del área de estudio corresponde a bosque tropical seco, el cual presenta un dosel que no rebasa los $15 \mathrm{~m}$ de altura en su etapa tardía y que del $50-75 \%$ de sus especies arbóreas pierden las hojas durante la época seca. Entre las especies reportadas se pueden mencionar a: 
Bursera simaruba, Guaiacum sanctum, Haematoxylum campechianum, Lonchocarpus yucatanensis, Maytenus schippi, y Thouinia paucidentata y con asociaciones secundarias las especies Lonchocarpus xuul, Lysiloma latisiliquium y Metopium brownei (Martínez-Salas \& Galindo-Leal, 2002).

Descripción del manejo: Se aplicaron entrevistas semiestructuradas a más del $60 \%$ de los pobladores de las dos comunidades que realizan manejo de la vegetación secundaria (46 en total). Las entrevistas se hicieron para describir el proceso y las fases que se siguen en el manejo de esta vegetación.

Diseño de muestreo: En las 46 entrevistas semiestructuradas realizadas para caracterizar el manejo se incluyeron preguntas para conocer la historia de uso de las parcelas. Con base en las entrevistas, se seleccionaron quince parcelas de vegetación secundaria de 15-19 años con las siguientes condiciones: cinco con manejo apícola (MA), cinco con manejo forestal (MF), cinco parcelas de vegetación secundaria sin manejo (SM), y cinco parcelas de bosques tropicales secos (BTS).

\section{Composición florística, diversidad} de especies y estructura de la vegetación arbórea: Se trazaron parcelas circulares de $1000 \mathrm{~m}^{2}$, con un círculo interior de $400 \mathrm{~m}^{2}$. En los círculos de $400 \mathrm{~m}^{2}$ se identificaron, etiquetaron, contaron y midieron todos los árboles de especies leñosas de $>1.30 \mathrm{~m}$ de altura y con DAP $>2.5 \mathrm{~cm}$. En el círculo de $1000 \mathrm{~m}^{2} \mathrm{se}$ identificaron, etiquetaron, contaron y midieron todos los individuos con altura de $>1.30 \mathrm{~m}$ y DAP >10 cm (adaptado de SARH, 1994; Programa Mexicano del Carbono, 2011).

La identificación de especies arbóreas se realizó con la ayuda de dos parataxónomos (Demetrio Álvarez Montejo y Manuel Arana), apoyados con el listado de Martínez-Salas, Sousa-Sánchez y Ramos-Álvarez (2001). Se corroboró la nomenclatura científica con la página del Missouri Botanical Garden (Tropicos, 2013) y The Plant List (Theplantlist,
2013). Se construyó el listado florístico con los datos de especie, género y familia botánica.

Para analizar la similitud entre las parcelas de las diferentes condiciones, se realizó un análisis de agrupamiento (dendrograma), utilizando el índice de similitud de Bray y Curtis (1957), y el método de agrupamiento de pares promedio sin ponderación (UPGMA) a partir de la matriz de similitud. Las variables utilizadas fueron el número de individuos registrado por cada una de las especies detectadas en las condiciones.

Se calculó la riqueza específica y se obtuvo la diversidad de especies en las cuatro condiciones de estudio, utilizando los índices de Shannon- Wiener, $\left(\mathrm{H}^{\prime}=-\Sigma \mathrm{pi}^{*} \ln \mathrm{i}\right.$; Magurran, 2004) y el número equivalente de especies $\left(\mathrm{D}=\exp -\sum \mathrm{p}_{\mathrm{i}} \ln \mathrm{p}_{\mathrm{i}}\right)$. El número equivalente de especies sirve para dar mayor robustez a los resultados de diversidad (Jost, 2006; Jost \& González-Oreja, 2012).

La estructura de la vegetación se analizó a partir de la distribución de frecuencias de clases diamétricas en un histograma, distinguiendo cinco intervalos: $\leq 5 \mathrm{~cm},>5 \mathrm{~cm}$ a $10 \mathrm{~cm},>10$ $\mathrm{cm}$ a $20 \mathrm{~cm},>20 \mathrm{~cm}$ a $30 \mathrm{~cm},>30 \mathrm{~cm}$.

Se estimó la densidad promedio por condición (indiv./ha; Krebs, 1999) y el área basal promedio $\left(\mathrm{m}^{2} / \mathrm{ha}\right)$, donde $\mathrm{AB}=(\mathrm{DAP} / 2)^{2} \pi$; DAP es el diámetro a la altura del pecho y $\pi=$ 3.1416 (Brower, Zar, \& Von-Ende, 1997). La importancia relativa de las especies por condición, (VIR) se calculó como la sumatoria de la abundancia relativa (número de individuos por especies/número total de individuos de todas las especies*100), la frecuencia relativa (frecuencia de una especie/suma de la frecuencia de todas las especies*100), y área basal relativa (área basal de cada especie/área basal total de todas las especies*100; Mueller-Dombois \& Ellemberg, 2002).

Se realizó un análisis de similitud (ANOSIM), utilizando la distancia del índice de Bray Curtis (Clarke, 1993; Magurran, 2004), para examinar diferencias en la composición de especies entre las diferentes condiciones. Este análisis no paramétrico estima una medida absoluta de distancia entre los grupos (R), 
que toma valores entre 0 y 1 , mientras más se acerca a cero, mayor es la similitud entre las muestras. El nivel de significancia se calculó mediante la prueba de Bonferroni, que permite controlar el nivel de confianza simultáneo para un conjunto completo de intervalos de confianza, es decir el error de tipo I. Para estimar la proporción que aporta cada especie a la diferencia entre condiciones, determinada por el porcentaje de disimilitud entre ellas, se empleó la subrutina SIMPER (Clarke, 1993). Estos análisis se realizaron con el programa PAST 2.17c (Hammer, Harper, \& Ryan, 2001).

Se aplicó un análisis de normalidad (Prueba de Levin) a las variables número de familias, riqueza de especies, densidad y área basal. Se realizó un análisis de varianza unifactorial (ANOVA) para determinar si había diferencias significativas entre las diferentes condiciones (MA, MF, SM, BTS) para las variables que cumplieron los supuestos de la prueba (riqueza de especies, área basal y densidad). Cuando se encontraron diferencias significativas se realizaron comparaciones múltiples con la prueba de Tukey, $\alpha=0.05$ (Zar, 2010). En el caso del número de familias, la comparación se realizó mediante la prueba de KruskalWallis, por no cumplir con los supuestos del ANOVA. Para estos análisis se empleó el programa Statistica 2007.

Los valores del índice de Shannon se compararon con una prueba de $\mathrm{t}$ de Student modificada por Hutcheson (1970), para probar diferencias significativas $(\mathrm{p}<0.05)$ entre pares de condiciones (MA - MF, MA - MS, MA BTS, MF - SM, MF - BTS y SM - BTS).

\section{RESULTADOS}

Descripción del manejo: Los 46 entrevistados describieron el manejo forestal dividido en cuatro etapas y dirigido al aprovechamiento forestal (MF) o apícola (MA). La primera etapa consiste en la selección de parcelas con vegetación secundaria en diferentes fases $(4 \mathrm{a}$ 25 años) para el manejo, esto depende de las condiciones en las cuales tienen sus parcelas. Durante la segunda fase se realiza el aclareo o deshierbe, corte de ramas, arbustos, lianas, bejucos y eliminación de árboles suprimidos. En las entrevistas, los propietarios mencionaron que al realizar el aclareo, dejan las especies de su interés para obtener a corto, mediano y largo plazo, recursos del área manejada, sin eliminar especies de manera selectiva. Sin embargo, los resultados de diversidad en este trabajo demuestran que si hay una extracción selectiva de especies en la condición MA, pues es significativamente menor que en el resto de las condiciones.

Por otro lado, comentaron que durante la fase de aclareo se cortaron pocos individuos y no se extrajeron recursos del área (ramasrebrotes, árboles pequeños, torcidos o suprimidos), quedando todo en el sitio. Sin embargo, cuando se realizaron los muestreos, se observó que las parcelas con manejo forestal (MF) presentaban menos individuos que las parcelas de manejo apícola (MA). Esto concuerda con los resultados de los análisis estadísticos en los cuales las densidades entre MA y MF fueron significativamente diferentes. Esto sugiere que hay una extracción selectiva de individuos en el caso del MF.

La tercera etapa consiste en el enriquecimiento o siembra de especies para su aprovechamiento comercial como Pimenta dioica (pimienta - 14 entrevistados), Brosimum alicastrum (ramón - 40 entrevistados), Manilkara zapota (zapote - 34 entrevistados), Swietenia macropylla (caoba - 5 entrevistados), y Cedrela odorata (cedro - 6 entrevistados). Los ejidatarios señalaron que las especies sembradas durante el enriquecimiento producirían un ingreso económico a mediano y largo plazo, al aprovechar los frutos de la pimienta para condimentos; del ramón las hojas para forraje, y sus frutos y semillas para harinas; del zapote el látex para elaborar goma de mascar natural y los frutos que son comestibles, y del cedro y la caoba madera para comercializar. Finalmente, la cuarta fase consiste en el mantenimiento de vegetación secundaria.

Composición de especies: Se registraron 4490 individuos en total, pertenecientes 
a 101 especies, 79 géneros y 35 familias. En la MA se encontraron 56 especies, 49 géneros y 27 familias, la MF con 65 especies, 55 géneros y 30 familias, la SM con 63 especies, 51 géneros y 27 familias y el BTS con 73 especies, 60 géneros y 33 familias (Apéndice 1). El número de familias entre las parcelas de estudios por condiciones (MA, MF, SM y BTS) no mostró diferencias significativas (Kruskal-Wallis, $\mathrm{p}=0.4677$ ). Las familias con mayor número de especies en las cuatro condiciones fueron Fabaceae (14), Euphorbiaceae (6), Polygonaceae (5), Boraginaceae, Rubiaceae y Rutaceae (4), Myrtaceae, Salicaceae, Sapindaceae y Sapotaceae (3), aportando en su conjunto más del $50 \%$ del total de especies registradas (Apéndice 1).

Las especies más abundantes en las parcelas de estudio fueron Lonchocarpus guatemalensis, Lonchocarpus yucatanensis, Esenbeckia berlandieri, Bursera simaruba, Pilocarpus racemosus y Drypetes lateriflora.

Las SM y los BTS fueron las condiciones con mayor número de especies en común $(50,57.47 \%)$. Entre la vegetación secundaria manejada (MA y MF) se compartieron 45 $(59.21 \%)$ especies en total, encontrando que Sideroxylon salicifolium se presentó solamente en estas condiciones. La SM compartió con MA $43(56.57 \%)$ y con el MF 45 (54.21\%) especies. Con respecto al BTS, la MA y la MF comparten $46(55.42 \%)$ y $47(51.64 \%)$ especies, respectivamente.

El análisis de similitud permitió reconocer la formación incipiente de grupos $(30 \%$ de similitud) asociados con las diferentes condiciones. El grupo "a" está conformado por el BTS2 que se encuentra separado de los otros grupos. El grupo "b" se conforma por MF y SM. En el grupo "c" se encuentran los BTS y SM, agrupando al $80 \%$ de las parcelas de los BTS. El grupo "d" está conformado por todas las parcelas de MA, algunas parcelas de MF y SM (Fig. 1). El ANOSIM mostró que la composición de especies entre las condiciones fue estadísticamente diferente $(\mathrm{R}=0.3657$, $\mathrm{p}=0.0005)$. Las diferencias se encontraron entre MA y BTS $(\mathrm{R}=0.812, \mathrm{p}=0.0426)$, y MF y BTS $(R=0.504, p=0.048)$. Mientras que SM no presentó diferencias significativas respecto al resto de las condiciones MA, MF y BTS. El análisis SIMPER permitió identificar que las condiciones MA-BTS, presentaron una disimilitud promedio del $81.46 \%$. Las especies que contribuyeron a estas diferencias se presentan en el cuadro 1.

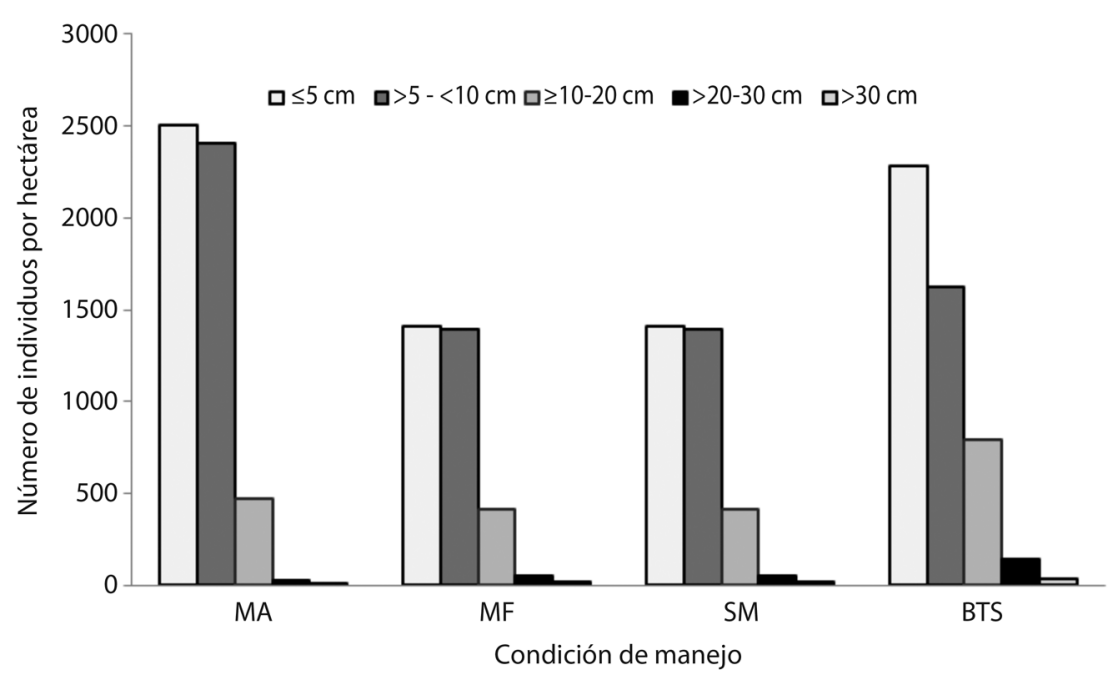

Fig. 1. Distribución de individuos por clase diamétrica en cuatro condiciones de bosque.

Fig. 1. Distribution of individuals per diameter classes in four forest conditions. 
CUADRO 1

Especies que contribuyen con los mayores porcentajes a la disimilitud encontradas en MA-BTS y MF-BTS

TABLE 1

Species contributing percentages greater than the dissimilarity found in MA-BTS and MF-BTS

\begin{tabular}{lccc}
\multicolumn{1}{c}{ Especie } & MA-BTS & & \% Contr. A \\
\hline Lonchocarpus guatemalensis & Pd & \% Contr. & 25.9 \\
Pilocarpus racemosus & 21.1 & 25.9 & 35.68 \\
Lonchocarpus yucatanensis & 7.97 & 9.79 & 43.25 \\
Nectandra salicifolia & 6.16 & 7.56 & 49.9 \\
Drypetes lateriflora & 5.42 & 6.65 & 54.51 \\
\multicolumn{1}{r}{ Especie } & 3.76 & 4.61 & Contr. A \\
\multicolumn{1}{c}{ MF-BTS } & & 13.47 \\
Pilocarpus racemosus & Pd & Contr. & 21.42 \\
Lonchocarpus yucatanensis & 9.66 & 13.47 & 27.76 \\
Drypetes lateriflora & 5.71 & 7.96 & 33.85 \\
Lonchocarpus guatemalensis & 4.55 & 6.34 & 39.00 \\
Neomillspaughia emarginata & 4.37 & 6.09 & 43.70 \\
Esenbeckia berlandieri & 3.70 & 5.15 & 47.17 \\
Bursera simaruba & 3.38 & 4.70 & 50.43 \\
Amyris elemifera & 2.48 & 3.46 & 3.27 \\
\hline
\end{tabular}

Resultados obtenidos con el análisis SIMPER. Promedios de disimilitud (Pd); porcentaje de contribución (\% Contr.); porcentaje de contribución acumulado (\% Contr. A).

Results obtained with SIMPER analysis. Average dissimilarity (Pd); percentage contribution (\% Contr.); cumulative contribution percentage ( $\%$ Contr. A).

Diversidad de especies: El ANOVA muestra que la riqueza de especies entre las condiciones no mostró diferencias significativas $(p=0.2762)$. La prueba $t$ de Student con una $\mathrm{p}<0.05$, determinó que hay diferencias significativas entre la diversidad de especies de la MA con respecto a las condiciones MF $(\mathrm{t}=$ -11.789 , g.l = 2 013.4), SM ( $\mathrm{t}=-12.934$, g.l. $=$ 2 252.7) y BTS $(\mathrm{t}=-14.125$, g.l. $=2$ 326). Por otro lado, la diversidad de especies medida con el número equivalente de especies en la MA fue menor al $50 \%$ respecto de MF, SM y BTS (Cuadro 2).

Estructura: Las distribuciones del número de individuos por clases diamétricas tuvieron en general un comportamiento semejante al de una $\mathrm{J}$ invertida. Las distribuciones de las clases diamétricas en las dos primeras clases $(\leq 5 \mathrm{y}>$ 5-10 cm) fueron en todas las condiciones las más abundantes, con $86.73 \%$ de individuos. La tercera clase diamétrica $(>10-20 \mathrm{~cm})$ presentó el $11.41 \%$ de individuos, siendo el BTS y MF las condiciones que sobresalen con el mayor y menor número de individuos, respectivamente. La cuarta clase diamétrica $(20-30 \mathrm{~cm})$ estuvo representada con el $1.55 \%$ de individuos, en el que el BTS y la SM presentaron los valores más altos de individuos. La última clase diamétrica $(>30 \mathrm{~cm})$ presentó la menor cantidad de individuos $(0.3 \%)$ de todas las clases, y el BTS seguido de la MF concentraron el mayor número de individuos en esta categoría (Fig. 2).

De acuerdo con los resultados del ANOVA, al comparar la densidad promedio entre las cuatro condiciones estudiadas, se observó una diferencia significativa entre ellas $(p=0.027)$. Las diferencias se presentaron entre las condiciones MA y MF (Tukey; $p=0.029$ ). La MA presentó una densidad promedio de individuos 
CUADRO 2

Diversidad de especies, densidad y área basal promedio en las cuatro condiciones de estudio

TABLE 2

Species diversity, mean density and basal area in the four studied conditions

\begin{tabular}{ccccc} 
Condiciones & Shannon-Wiener, H' & Número equivalente de especies, D & $\bar{X}$ de la densidad (ind./ha) & $\bar{X}$ del área basal $\left(\mathrm{m}^{2} / \mathrm{ha}^{\prime}\right)$ \\
MA & 2.36 & 10.59 & $5413 \pm 770.26^{*}$ & $12.67 \pm 4.71^{\text {n.s }}$ \\
MF & 3.13 & 22.82 & $3289 \pm 1183.60^{*}$ & $13.47 \pm 2.50^{\text {n.s }}$ \\
SM & 3.13 & 22.87 & $5148 \pm 1162.07^{\text {n. s }}$ & $14.82 \pm 5.25^{\text {n.s }}$ \\
BTS & 3.22 & 25.00 & $4874 \pm 1107.45^{\text {n.s }}$ & $24.89 \pm 1.56^{* *}$ \\
\hline
\end{tabular}

Significancia $*=\leq 0.05, * *=0.001, \mathrm{n} . \mathrm{s} .=$ no significativo.

Significance $*=<0.05, * * \leq 0.001, \mathrm{n}$. s. $=$ no significant differences.

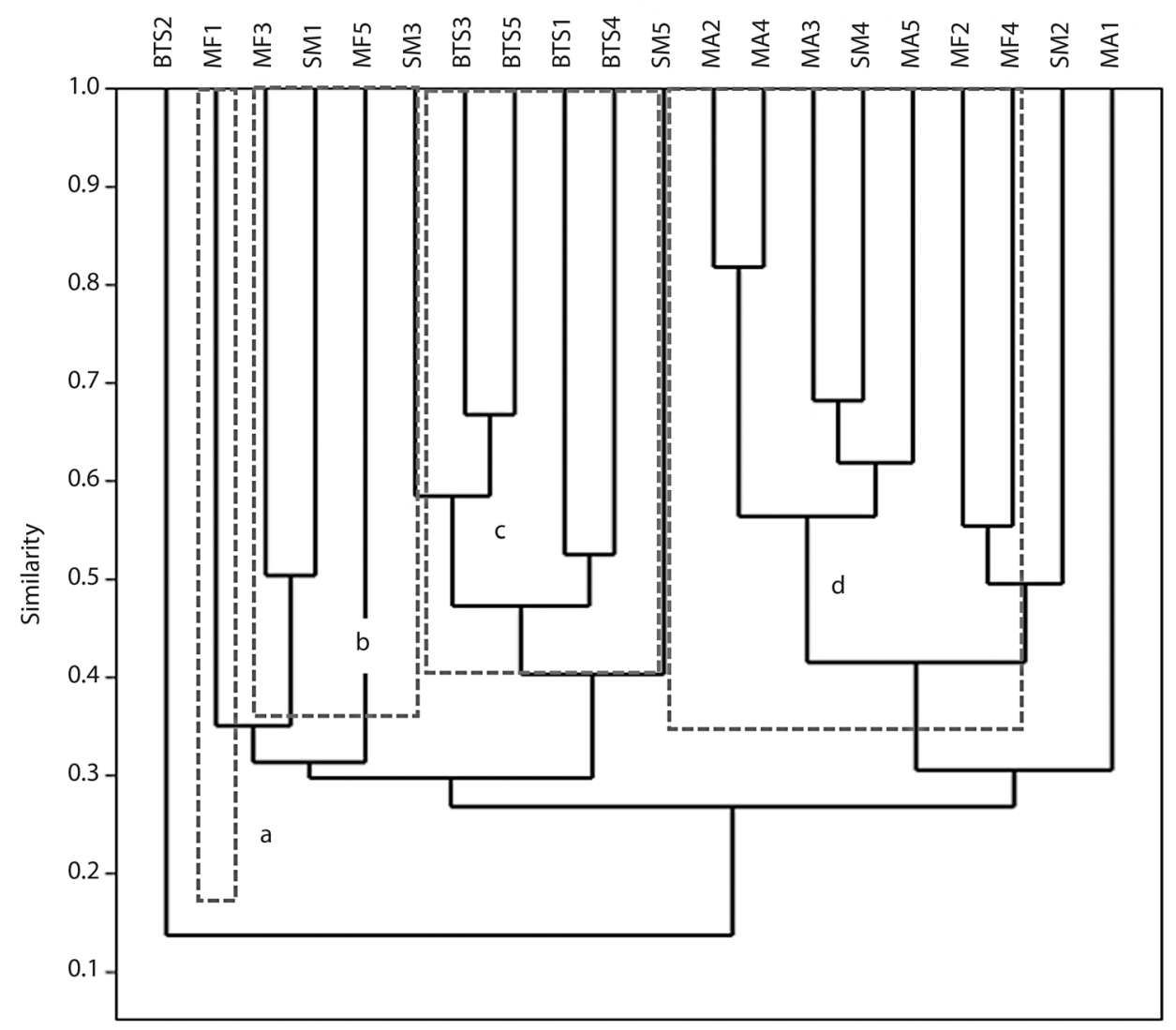

Fig. 2. Análisis de agrupamiento entre las parcelas estudiadas en Puebla de Morelia (PM) y Kilómetro 120 (Km 120), con una significancia de $(\mathrm{p}<0.05)$. Las letras $\mathrm{a}, \mathrm{b}, \mathrm{c}$ y d, dentro de los rectángulos grises con líneas discontinuas representan los cuatro grupos que se forman.

Fig. 2. Cluster analysis between plots of Puebla of Morelia (PM) and Kilómetro 120 (Km 120), with a significance of $(\mathrm{p}<0.05)$. The letters $\mathrm{a}, \mathrm{b}, \mathrm{c}$ and $\mathrm{d}$, in gray rectangles with dashed lines represent the four groups formed. 
significativamente mayor (5413 \pm 770.26 ind./ ha) que la MF (3289 \pm 1183.60 ind./ha). Al aplicar el ANOVA, el área basal promedio entre las cuatro condiciones mostró diferencias significativas $(\mathrm{p}=0.0004)$. Siendo la condición BTS la que presentó un área basal promedio significativamente mayor que el resto de las condiciones MA (Tukey; $\mathrm{p}=0.0007$ ), MF (Tukey; $\mathrm{p}$ $=0.001)$ y SM (Tukey; $p=0.003)$ (Cuadro 2).

De las 10 especies con mayor VIR, MA y MF compartieron seis especies, MA y SM (seis), MA y BTS (cinco), MF y SM (seis), MF y BTS (cuatro), y en la SM y BTS (siete). En el MA la especie con mayor VIR fue Lonchocarpus guatemalensis, en MF Bursera simaruba, en SM y en BTS Lonchocarpus yucatanensis (Apéndice 2).

\section{DISCUSIÓN}

El manejo forestal que se realiza en las comunidades estudiadas emplea cuatro fases y está enfocado al aprovechamiento apícola y forestal de vegetación secundaria derivada de la transformación de BTS en parcelas agrícolas, en las cuales se aplica extracción selectiva y enriquecimiento con especies. De acuerdo con el manejo tradicional, las SM en algún momento volverían a incorporarse al ciclo de la agricultura tradicional; mientras que el manejo estudiado en las parcelas de MA y MF tiene como fin obtener recursos maderables y no maderables, por lo que se establecerán como áreas forestales permanentes en uso. La estrategia de manejo de vegetación secundaria estudiada permite disminuir la presión sobre los bosques maduros, ya que la provisión de productos maderables y no maderables se focaliza en la vegetación secundaria. El uso doméstico y la venta de los productos derivados del manejo estudiado, generaría un ingreso a la economía familiar de los ejidatarios.

Las familias de plantas, con mayor número de especies en este estudio coinciden con las 10 familias más frecuentes y con mayor número de especies reportadas para la Península de Yucatán (Carnevali-Fernández Concha, Tapia-Muñoz, Duno de Stefano, \&
Ramírez-Morillo, 2010). También concuerdan con las reportadas en otros estudios de BTS y vegetación secundaria del neotrópico (RomeroDuque, Jaramillo, \& Pérez-Jiménez, 2007; Gutiérrez-Báez, Ortíz-Díaz, Flores-Guido, \& Zamora-Crescencio, 2012), en BTS sujetos a algún tipo corta selectiva (Rendón-Carmona, Martínez-Yrízar, Balvanera, \& Pérez-Salicrup, 2009) y en su vegetación secundaria (ZamoraCrescencio et al., 2011).

Las especies identificadas en el estudio tienen usos melíferos y forestales, independientemente de la condición en la cual se encontraron. Que las condiciones estudiadas de vegetación secundaria (MA, MF, SM) compartan más del $50 \%$ de especies, se explica en parte porque las áreas para aprovechamiento apícola y forestal forman parte de un mosaico heterogéneo donde las diferentes condiciones estudiadas se entremezclan. Este arreglo espacial permite el intercambio de germoplasma, y la presencia de árboles cortados que rebrotan (tocones) aporta elementos maduros que homogeneizan la composición (Martínez-Ramos \& García-Orth, 2007).

Por otro lado, en el dendrograma se observó que todas las condiciones son muy heterogéneas, presentando una baja similitud entre sí (30\%). Este comportamiento se debe probablemente a distintos factores, por ejemplo, la posición topográfica de la parcela de muestreo BTS2 que conforma al grupo "a" la hizo ser diferente en composición que las demás unidades de BTS, porque está ubicada en una loma, mientras las demás parcelas se ubicaron en planicies. Lo anterior puede sumarse a factores de manejo. Por ejemplo, para el grupo "b" (parcelas de MF y SM) y el grupo "d" (parcelas de MA, unas de MF y SM), la variación puede estar reflejando las decisiones que toma cada propietario en cuanto a qué especies dejan o eliminan al realizar el manejo, aun cuando tengan el mismo objetivo. Por último, el contexto del paisaje puede agregar efectos distintivos en la comunidad que surge en la vegetación arbórea subsecuente a la tala del bosque, esto puede explicar el grupo "c" (mayoría de BTS y unas parcelas de $\mathrm{SM}$ ). La vegetación secundaria 
sin manejo, al estar cercanas a los bosques maduros, recibe semillas de los bosques en mayor medida que aquellas alejadas de fragmentos conservados de bosque (Guariguata $\&$ Ostertag 2002).

Mientras que las diferencias en la composición de especies entre MA, MF y BTS pueden atribuirse a especies características en una edad o etapa sucesional (Liebsch, Goldenberg, \& Mendes-Marques, 2007), o probablemente al manejo que están realizando los propietarios de las parcelas. Las especies que aportaron el porcentaje más alto de disimilitud entre la vegetación secundaria manejada y el BTS en este estudio, son reportadas como especies indicadoras de áreas donde hay influencia antropogénica o de bosques maduros, respectivamente; además de tener un uso frecuente como especies melíferas o forestales (Martínez-Salas \& Galindo-Leal, 2002; Arellano-Rodríguez, Flores-Guido, Tun-Garrido, \& Cruz-Bojórquez, 2003; Herbario CICY, 2010).

Según investigaciones previas sobre el tema, los disturbios antropogénicos, como el aclareo, reducen la riqueza y diversidad de especies (Brown \& Gurevitch, 2004; Kumar \& Shahabuddin, 2005; Clark \& Covey, 2012). En este trabajo, por el contrario, la riqueza de especies no presentó diferencias significativas entre vegetación manejada y sin manejo por efecto del aclareo. Probablemente por la cercanía entre las diferentes condiciones lo que favorece el intercambio de germoplasma $\mathrm{y}$, por tanto, la homogeneidad de especies entre condiciones.

La diversidad de especies en MA disminuyó con respecto a las demás condiciones (MF, SM y BTS), coincidiendo con lo reportado en un BTS que fue sujeto a extracción selectiva en diferentes ciclos de corta (Rendón-Carmona et al., 2009). La disminución de la diversidad en la MA probablemente se deba a una extracción selectiva de especies que no son útiles para la producción de miel, para favorecer la presencia de especies que incrementan la producción de este producto.

El compartimiento semejante a una "J invertida" en las distribuciones de clases diamétricas, indica que la vegetación mantiene procesos de recambio natural. Estos recambios garantizan el reemplazo de árboles que son eliminados por diversas causas (García-Licona, Esparza-Olguín, \& Martínez-Romero, 2014), en el caso particular de este trabajo durante el manejo y aprovechamiento.

Que la densidad promedio de los individuos de MA y MF no fuera significativamente menor respecto a la SM, puede asociarse a que la extracción de individuos durante el aclareo fue baja en ambas condiciones. Mientras que la diferencia entre MA y MF puede asociarse a que en el MF se extrajeron más individuos, al tratar de eliminar competencia y permitir el paso de luz para que las especies de interés, se desarrollen más rápidamente para su aprovechamiento a mediano y largo plazo (Hutchinson, 1993; Guariguata, 1999). Otra explicación es el tipo de aprovechamiento inmediato que le dan a los recursos forestales (producción de leña, palancas, cercos vivos, entre otros).

El que las áreas basales promedio de MA, MF y SM no presentaran diferencias significativas, puede deberse a que no ha transcurrido suficiente tiempo para que el manejo forestal, permita observar un crecimiento notable en los diámetros de los individuos. Como se esperaba los BTS presentaron valores de área basal mayor que la vegetación secundaria manejada y no manejada (Lebrija-Trejos, Bongers, Pérez-García, \& Meave, 2008; Lebrija-Trejos, Meave, Poorter, Pérez-García, \& Bongers, 2010; Aryal, de Jong, Ochoa-Gaona, Esparza-Olguín, \& Mendoza-Vega, 2014). Es de esperar que a mediano plazo los diámetros se incrementen en las MA y MF acercándose a la condición de los BTS.

Las especies con los valores más altos de VIR en este trabajo, han sido reportadas como algunas de las que presentan mayor VIR en bosque tropical seco y su vegetación secundaria en la Península de Yucatán (White \& Hood, 2004; Palmer-Dickson, 2009; Schmook, 2010; Zamora-Crescencio et al., 2011). La dominancia de estas especies podría deberse al uso que se les da en el presente, por ejemplo L. guatemalensis es una especie con alto uso melífero 
(VIR mayor en MA), mientras B. simaruba es usada para cerco vivo, forraje para ganado, madera para palillo, (dominante en MF).

Al evaluar el impacto del manejo forestal dirigido en las áreas de MA y MF, se concluye que las decisiones del productor aunque probablemente modifican la composición de especies entre unidades, incluso del mismo tipo; no impiden que la riqueza y diversidad (MF) se conserven en el paisaje. Las diferencias en la diversidad de especies y densidad de individuos que se observan entre algunas condiciones, sugieren que es importante mantener el monitoreo de las áreas manejadas para detectar efectos del manejo, que puedan ser adversos o favorables para la conservación de la diversidad florística del BTS.

\section{AGRADECIMIENTOS}

Los autores agradecen a Demetrio Álvarez y Manuel Arana por su apoyo en la identificación de las especies y a los propietarios de las parcelas: Alcides Pérez, Reyna Pérez, Zacarías Pérez, Pablo Gómez, José Luis Ramos, Macario Hernández, Taurino Durán, Florencio Castillo, Elvia Olán, Ezequías Arcos, Joaquín Torres, Filemón Pérez, quienes nos permitieron el acceso. El Proyecto Monitoreo Adaptativo de la Reserva de la Biosfera de Calakmul (FMCN) MAREBICA, que financió el trabajo de campo. El Consejo Nacional de Ciencia y Tecnología que otorgó la beca número 270023 a la primera autora. El Colegio de la Frontera Sur porque otorgó un apoyo económico e infraestructura para llevar a cabo esta investigación.

\section{RESUMEN}

El bosque tropical seco (BTS) de la Península de Yucatán ha sido manejado por siglos, pero la relación del efecto del manejo sobre la diversidad de árboles no ha sido completamente entendida. El objetivo de este estudio fue evaluar el efecto del manejo forestal (aclareos, aprovechamiento y enriquecimiento de especies) en la estructura de la vegetación secundaria derivada de bosques tropicales secos, en dos comunidades en Calakmul, Campeche, Sureste de México. Se analizaron cambios en la composición, riqueza de especies, diversidad de especies y estructura en vegetación secundaria sujetas a los siguientes tipos de manejo: 1) vegetación secundaria con manejo apícola (MA), 2) vegetación secundaria con manejo forestal (MF), 3) vegetación secundaria sin manejo (SM) y bosque tropical seco (BTS). La composición de especies fue similar entre vegetación secundaria manejada y no manejada. Por otro lado, entre vegetación secundaria manejada y el BTS hubo diferencias en la composición de especies. La riqueza de especies no fue diferente entre todas las condiciones. La MA mostró la más baja diversidad de especies y presentó la mayor densidad promedio (5 $413 \pm 770.26$ ind./ha). La MF tuvo la menor densidad promedio (3 $289 \pm 1183.60$ ind./ ha). El BTS mostró la mayor área basal promedio (24.89 $\pm 1.56 \mathrm{~m}^{2} / \mathrm{ha}$ ) respecto a las demás condiciones. Se concluye que es necesario mantener el monitoreo de las áreas manejadas, para detectar efectos del manejo que pueden ser adversos o favorables para la conservación de la diversidad florística de los BTS.

Palabras clave: manejo apícola, manejo forestal, bosque seco, estructura, composición florística.

\section{REFERENCIAS}

Arellano-Rodríguez, J. A., Flores-Guido, J. S., Tun-Garrido, J., \& Cruz-Bojórquez, M. M. (2003). Nomenclatura, forma de vida, uso, manejo y distribución de las especies vegetales de la Península de Yucatán. México: Universidad Autónoma de Yucatán.

Aryal, D. R., de Jong, B. H. J., Ochoa-Gaona, S., EsparzaOlguín, L., \& Mendoza-Vega, J. (2014). Carbon stocks and changes in tropical secondary forests of southern Mexico. Agriculture, Ecosystems and Environment, 195, 220-230.

Beckerman, S. (1987). Swidden in Amazonia and the Amazon rim. In B. L. Turner \& S. B. Brush (Eds.), Comparative Farming Systems (pp. 55-94). New York: Guilford Press.

Bray, J. R., \& Curtis, J. T. (1957). An ordination of the upland forest communities of Southern Wisconsin. Ecological Monographs, 27, 326-349.

Brower, J. E., Zar, J. H., \& Von-Ende, C. N. (1997). Field and laboratory methods for general ecology. Boston, USA: WCB-McGraw-Hill.

Brown, K., \& Gurevitch, J. (2004). Long-term impacts of logging on forest diversity in Madagascar. Proceedings of the National Academy of Sciences, 101(16), 6045-6049.

Consejo Nacional de Evaluación de la Política de Desarrollo Social (CONEVAL). (2012). Campeche 20102012. Recuperado de http://www.coneval.gob.mx/ InformesPublicaciones/Documents/folletos_pobrezayrezagosocial/campeche_pob_rez_soc.pdf 
Consejo Nacional de Población (CONAPO). (2010). Índice de Marginación por entidad federativa y municipio. Recuperado de http://www.conapo.gob.mx/en/ CONAPO/Indices_de_Marginacion_2010_por_entidad_federativa_y_municipio

Carnevali-Fernández Concha, G., Tapia-Muñoz, J. L., Duno de Stefano, R., \& Ramírez-Morillo, I. M. (Eds.). (2010). Flora Ilustrada de la Península de Yucatán: Listado Florístico. México: Editorial Centro de Investigación Científica de Yucatán.

Clarke, K. R. (1993). Non-parametric multivariate analyses of changes in community structure. Australian Journal Ecology, 18, 117-143.

Clark, J. A., \& Covey, K. R. (2012). Tree species richness and the logging of natural forests: A meta-analysis. Forest Ecology and Management, 276, 146-153.

Fujii, S., Kubota, Y., \& Enoki, T. (2009). Resilience of stand structure and tree species diversity in subtropical forest degraded by clear logging. Journal of Forest Research, 14, 373-387.

Galindo-Leal, C. (1999). La Gran Región de Calakmul, Campeche: Prioridades biológicas de conservación y propuesta de modificación de la Reserva de la Biosfera (Reporte final). México: World Wildlife Fund.

García-Licona, J. B., Esparza-Olguín, L. G., \& MartínezRomero, E. (2014). Estructura y composición de la vegetación leñosa de selvas en diferentes estadios sucesionales en el ejido el Carmen II, Calakmul, México. Polibotánica, 38, 1-26.

Gillespie, T. W., Grijalva, A., \& Farris, C. N. (2000). Diversity, composition, and structure of tropical dry forests in Central America. Plant Ecology, 147, 37-47.

Guariguata, M. R. (1999). Early response of selected tree species to liberation thinning in a young secondary forest in Northeastern Costa Rica. Forest Ecology and Management, 124, 255-261.

Guariguata, M. R. \& Ostertag, R. (2002). Sucesión secundaria. In M. R. Guariguata \& G. H. Kattan (Eds.), Biología y conservación de bosques neotropicales (pp. 591-624). Costa Rica: Libro Universitario Regional.

Gutiérrez-Báez, C., Ortíz-Díaz, J. J., Flores-Guido, J. S., \& Zamora-Crescencio, P. (2012). Diversidad, estructura y composición de las especies leñosas de la selva mediana subcaducifolia del punto de unión territorial (Put) de Yucatán, México. Polibotánica, 33, 151-174.

Hammer, Ø., Harper, D. A. T., \& Ryan, P. D. (2001). PAST 2.17c: Paleontological Statistics Software Package for Education and Data Analysis. Recuperado de http://nhm2.uio.no/norlex/past/download.html

Herbario Centro de Investigación Científica de Yucatán (Herbario CICY). (2010). Flora Digital: Península de Yucatán. Recuperado de http://www.cicy.mx/ sitios/flora\%20digital/

Hutcheson, K. (1970). A test for comparing diversities based on the Shannon formula. Journal of Theorical Biology, 29, 151-154.

Hutchinson, I. (1993). Silvicultura y manejo en un bosque secundario tropical: caso Pérez Zeledón, Costa Rica. Revista Forestal Centroamericana, 2, 13-18.

Instituto Nacional de Estadística y Geografía (INEGI). (2009). Prontuario de información geográfica municipal de los Estados Unidos Mexicanos. Recuperado de http://www.inegi.gob.mx/prod serv/contenidos/espanol/bvinegi/productos/integracion/estados/ persc_estd/camp/Pers-cam.pdf

Instituto Nacional de Estadística y Geografía (INEGI). (2010). Censo de Población y Vivienda 2010. Recuperado de inegi.org.mx/sistemas/consulta resultados/ iter2010.aspx?c=27329\&s $=$ est

Instituto Nacional de Estadística y Geografía (INEGI). (2012). La apicultura en la Península de Yucatán. Censo agropecuario 2007. Recuperado de http:// www.inegi.org.mx/prod_serv/contenidos/espanol/ bvinegi/productos/censos/agropecuario/2007/agricola/peni_yuc_apicola/ApicYucatan.pdf

Jiménez-Osornio, J. J., Durán-García, R., Dupuy-Rada, J. M., \& González-Iturbe, J. A. (2010). Uso del suelo y vegetación secundaria en el Estado de Yucatán. In R. Durán \& M. Méndez (Eds.), Biodiversidad y Desarrollo Humano en Yucatán (pp. 460-464). México: CICY, PPD-FMAM, CONABIO, SEDUMA.

Jost, L. (2006). Entropy and Diversity. OIKOS, 113(2), 363-375.

Jost, L., \& González-Oreja, J. A. (2012). Midiendo la diversidad biológica: más allá del índice de Shannon. Acta Zoológica Lilloana, 56(1-2), 3-14.

Kalacska, M., Sanchez-Azofeifa, G. A., Calvo-Alvarado, J. C., Quesada, M., Rivard, B., \& Janzen, D. H. (2004). Species composition, similarity and diversity in three successional stages of a seasonally dry tropical forest. Forest Ecology and Management, 200, 227-247.

Kennard, D. K., Gould, K., Putz, F. E., Fredericksen, T. S., \& Morales, F. (2002). Effect of disturbance intensity on regeneration mechanisms in a tropical dry forest. Forest Ecology and Management, 162, 197-208.

Krebs, C. J. (1999). Ecological Methodology (2a edition). USA: Addison-Wesley Educational Publishers.

Kumar, R., \& Shahabuddin, G. (2005). Effects of biomass extraction on vegetation structure, diversity and composition of forests in Sariska Tiger Reserve, India. Environmental Conservation, 32(3), 248-259. 
Lawrence, D. (2004). Erosion of tree diversity during 200 years of shifting cultivation in Bornean rainforest. Ecological Applications, 14, 1855-1869.

Lawrence, D., D’Odorico, P., Diekmann, L., DeLonge, M., Das, R., \& Eaton, J. (2007). Ecological feedbacks following deforestation create the potential for a catastrophic ecosystem shift in tropical dry forest. Proceedings of the National Academy of Sciences of the United States of America, 104(52), 20696-20701.

Lebrija-Trejos, E., Bongers, F., Pérez-García, E. A., \& Meave, J. A. (2008). Successional Change and Resilience of a Very Dry Tropical Deciduous Forest Following Shifting Agriculture. Biotropica, 40(4), 422-431.

Lebrija-Trejos, E., Meave, J. A., Poorter, L., Pérez-García, E., \& Bongers, F. (2010). Pathways, mechanisms and predictability of vegetation change during tropical dry forest succession. Perspectives in Plant Ecology, Evolution and Systematics, 12, 267-275.

Leiva, J. A., Mata, R., Rocha, O. J., \& Gutiérrez-Soto, M. V. (2009). Cronología de la regeneración del bosque tropical seco en Santa Rosa, Guanacaste, Costa Rica. I. Características edáficas. Revista de Biología Tropical, 57(3), 817-836.

Liebsch, D., Goldenberg, R., \& Mendes-Marques, M. C. (2007). Florística e estrutura de comunidades vegetais em uma cronoseqüência de Floresta Atlântica no Estado do Paraná, Brasil. Acta Botanica Brasilica, 21, 983-992.

Magurran, A. E. (2004). Measuring biological diversity. Oxford, England: Blackwell Publishing.

Martínez-Ramos, M., \& García-Orth, X. (2007). Sucesión Ecológica y Restauración de las Selvas Húmedas. Boletín de la Sociedad Botánica de México, 80, 69-84.

Martínez-Salas, E., Sousa, M., \& Ramos-Álvarez, C. H. (2001). Listados florísticos de México. XXII. Región de Calakmul, Campeche (1 ${ }^{\mathrm{a}}$. Edición). México: Instituto de Biología, Universidad Nacional Autónoma de México.

Martínez-Salas, E., \& Galindo-Leal, C. (2002). La vegetación de Calakmul, Campeche, México: Clasificación, Descripción y Distribución. Boletín de la Sociedad Botánica de México, 71, 10-19.

Miranda, F. \& Hernández-X. E. (1963). Los tipos de vegetación de México y su clasificación. Boletín de la Sociedad Botánica de México, 28, 29-179.

Mueller-Dombois, D., \& Ellemberg, H. (2002). Aims and methods of vegetation ecology. USA: The Blackburn Press.
Murphy, P. G., \& Lugo, A. E. (1986). Ecology of tropical dry forest. Annual Review of Ecology and Systematics, $17,67-88$

Navarro-Martínez, M. A., Schmook, B., \& MartínezCastillo, J. (2000). Manejo tradicional de hubches en una comunidad maya de Quintana Roo. Foresta Veracruzana, 2(1), 19-30.

Ochoa-Gaona, S., Hernández-Vázquez, F., de Jong, B. H. J., \& Gurri-García, F. (2007). Pérdida de diversidad florística ante un gradiente de intensificación del sistema agrícola de roza-tumba-quema: un estudio de caso en la selva Lacandona, Chiapas, México. Boletín de la Sociedad Botánica de México, 81, 65-80.

Oliveira-Filho, A. T., Carvalho, D. A., Vilela, E. A., Curi, N., \& Fontes, M. A. L. (2004). Diversity and structure of the tree community of a fragment of tropical secondary forest of the Brazilian Atlantic Forest domain 15 and 40 years after logging. Revista Brasieira de Botánica, 27(4), 685-701.

Palmer-Dickson, R. (2009). Dry tropical forests recovery following smallholder cultivation in southern Yucatán. Rebeca. In R. Palmer-Dickson (autor), Secondary Diversity: Ecological and spectral dimensions of secondary succession following smallholder cultivation in the southern Yucatán. Worcester, Massachusetts: Clark University.

Programa Mexicano del Carbono. (2011). Manual de Campo para el Inventario Forestal Comunitario Cuantitativo. México: Colegio de Postgraduados.

Rendón-Carmona, H., Martínez-Yrízar, A., Balvanera, P., \& Pérez-Salicrup, D. (2009). Selective cutting of woody species in a Mexican tropical dry forest: Incompatibility between use and conservation. Forest Ecology and Management, 257, 567-579.

Romero-Duque, L. P., Jaramillo, V. J., \& Pérez-Jiménez, A. (2007). Structure and diversity of secondary tropical dry forests in México, differing in their prior land-use history. Forest Ecology and Management, $253,38-47$

Secretaría de Agricultura y Recursos Hidráulicos (SARH). (1994). Inventario Nacional Forestal Periódico 1992-1994. Recuperado de http://repositorio.inecc. gob.mx/ae2/ae_333.750972_m495-08i_1994.pdf

Schmook, B. (2010). Shifting Maize Cultivation and Secondary Vegetation in the Southern Yucatán: Successional Forest Impacts of Temporal Intensification. Regional Environmental Change, 10(3), 233-246.

Theplantlist.org (2013). The Plant List. Recuperado de http://www.theplantlist.org/

Tropicos.org. (2013). Missouri Botanical Garden. Recuperado de http://www.tropicos.org 
Vester, H. F., Lawrence, D., Eastman, J. R., Turner II, B. L., Calmé, S., Dickson, R., (...), \& Sangermano, F. (2007). Land Change in the Southern Yucatán and Calakmul Biosphere Reserve: Effects on Habitat and Biodiversity. Ecological Applications, 17(4), 989-1003.

White, D. A., \& Hood, C. S. (2004). Vegetation patterns and environmental gradients in tropical dry forests of the northern Yucatan Peninsula. Journal of Vegetation Science, 15, 151-160.
Zamora-Crescencio, P., Domínguez-Carrasco, M. del R., Villegas, P., Gutiérrez-Báez, C., Manzanero-Acevedo, L. A., Ortega-Haas, J. J., \& Puch-Chávez, R. (2011). Composición florística y estructura de la vegetación secundaria en el norte del estado de Campeche, México. Boletín de la Sociedad Botánica de México, 89, 27-35.

Zar, J. H. (2010). Biostatistical analysis. USA: Prentice-Hall. 
